\title{
A Further Result on the Cyclic Subspace
}

\author{
Huailei Wang \\ State Key Laboratory of Mechanics and Control for Mechanical Structures, Nanjing University of Aeronautics \\ and Astronautics, Nanjing, China \\ Email:whlay@nuaa.edu.cn
}

Received 28 February 2014; revised 28 March 2014; accepted 4 April 2014

Copyright ( 2014 by author and Scientific Research Publishing Inc.

This work is licensed under the Creative Commons Attribution International License (CC BY).

http://creativecommons.org/licenses/by/4.0/

(c) (i) Open Access

\begin{abstract}
Based on the geometric theories of vector space, a Cross-Identity theorem is proved for the relationship between the power kernels and power images of linear map $A-\lambda I$ on its cyclic subspace. By this result, a new approach of proof is found for the fact that a square matrix with only one eigenvalue and one-dimensional eigenspace is similar to a Jordan block matrix.
\end{abstract}

\section{Keywords}

Power Kernel, Power Image, Cyclic Subspace, Jordan Block Matrix, Linear Map

\section{Introduction}

Linear algebra has a wide range of application in most scientific and technological areas. As a fundamental part, the theory of vector space and linear map has developed to be a nearly perfect subject since nineteenth century. In the viewpoint of the author, when applying the theory to diverse problems, the more the detailed aspects of the knowledge one is in possession of, the more proficient one will become in dealing with those related problems. For example, in one of the excellent dynamical system monographs "Differential equations, Dynamical Systems, and an Introduction to Chaos" [1], the authors put a separate chapter named "Higher Dimensional Linear Algebra" to help the readers recall some relevant linear algebra knowledge needed for the analysis of differential equations and dynamical systems, despite so many linear algebra monographs available for reference, such as [2]-[8] to name a few. Although it is just a brief review of higher dimensional linear algebra, the authors presented therein a distinct method to prove that a $3 \times 3$ matrix can be transformed by a similarity transformation to a Jordan block matrix when it has only one eigenvalue and one-dimensional eigenspace. The essence of the method resides in a subtle analysis of the dimension of the kernel and the image of the matrix. Motivated by this method, this study is intended to generalize the results to the $n \times n$ matrix. For this purpose, it is found that it is necessary to gain a deep insight into some geometric properties of the $(\boldsymbol{A}-\lambda \boldsymbol{I})$-cyclic subspace, which finally leads to a more specific relationship discovered between the power- $m$ kernel and the power- $m$ image of 
linear map $\boldsymbol{A}-\lambda \boldsymbol{I}$, which is reffered to as the Cross-Identity theorem below. As a corollary of this theorem, we proved the fact that a $n \times n$ square matrix with only one eigenvalue and one eigenvector is similar to a Jordan block matrix.

The paper is organized as follows. In Section 2, some basic facts will be recalled as preliminaries for the good understanding of the work. Section 3 presents the main results of this paper with some lemmas preceding the Cross-Identity theorem. Some conclusions are drawn finally in Section 4.

\section{Preliminaries}

Let $V$ be a $n$-dimensional vector space over the complex numbers, and $L(V)$ be the set of all linear maps mapping the vector space $V$ into itself, and let Ker and Ima denote the kernel (null space) and the image (range) of a linear map $A \in L(V)$, respectively, then it is well known that if the characteristic polynomial of $\boldsymbol{A}$ is factorized as $f(\lambda)=\left(\lambda-\lambda_{1}\right)^{r_{1}}\left(\lambda-\lambda_{2}\right)^{r_{2}} \cdots\left(\lambda-\lambda_{s}\right)^{r_{s}}, \lambda_{1}, \lambda_{2} \cdots \lambda_{s}$ being its distinct eigenvalues, then the vector space $V$ can be decomposed into the direct sum of some subspaces, i.e. $V=V_{1} \oplus V_{2} \oplus \cdots V_{s}$, where $V_{i}=\operatorname{Ker}(\boldsymbol{A}-\lambda \boldsymbol{I})^{r_{i}}$ with $\boldsymbol{I} \in L(V)$ denoting the identity map. Moreover, it can also be proved by using the primary decomposition that each of the above subspace $V_{i}$, and hence the whole vector space $V$, can be further expressed as a direct sum of $\boldsymbol{A}$-invariant cyclic subspaces [3]. Because the transformation matrix of a linear map under a cyclic basis takes on a Jordan normal form, the above decomposition theories lead straightforward to a geometric approach of proof for the fact that any square matrix $\boldsymbol{A}$ can be brought into Jordan normal form through a similarity transformation with a change-of-basis matrix (another way leading to this result is through the elementary divisor theory [7]).

From the above viewpoint, the cyclic subspace of a vector space shall be an important concept in the field of linear algebra. For the convenience of reference, some preliminary concepts are presented below, for which one can also refer to [3].

Definition 2.1 Cyclic vector Let $\alpha$ be a complex number, let $v \in V, \quad v \neq 0$, then $v$ is called $(\boldsymbol{A}-\alpha \boldsymbol{I})$-cyclic if there exists an integer $r \geq 1$ such that $(\boldsymbol{A}-\alpha \boldsymbol{I})^{r} v=0$. The smallest positive integer $r$ having this property will then be called a period of $v$ relative to $(\boldsymbol{A}-\alpha \boldsymbol{I})$.

Definition 2.2 Cyclic space The vector space $V$ will be called cyclic if there exists some number $\alpha$ and an element which is $(\boldsymbol{A}-\alpha \boldsymbol{I})-$ cyclic and $v, \boldsymbol{A} v, \cdots \boldsymbol{A}^{r-1} v$ generate $V$.

Theorem 2.3 if $v \neq 0$ is $(\boldsymbol{A}-\alpha \boldsymbol{I})$-cyclic, with period $r$, then $v,(\boldsymbol{A}-\alpha \boldsymbol{I}) v, \cdots,(\boldsymbol{A}-\alpha \boldsymbol{I})^{r-1} v$ are linearly independent.

Definition 2.4 Cyclic basis if $v \neq 0$ is $(\boldsymbol{A}-\alpha \boldsymbol{I})$-cyclic, with period $r$, then

$\left\{v,(\boldsymbol{A}-\alpha \boldsymbol{I}) v, \cdots,(\boldsymbol{A}-\alpha \boldsymbol{I})^{r-1} v\right\}$ is called a cyclic basis or Jodan basis for the cyclic space $V$ it generates.

Definition 2.5 Jordan block matrix A matrix is called a Jordan block matrix if it has the same number $\lambda$ on the diagonal, 1 above the diagonal, and 0 everywhere else.

Theorem 2.6 Let $A$ be a linear map defined on a $n$-dimensional cyclic space $V$, then the associated matrix $\boldsymbol{A}$ with respect to the cyclic basis $\left\{v,(\boldsymbol{A}-\alpha \boldsymbol{I}) v, \cdots,(\boldsymbol{A}-\alpha \boldsymbol{I})^{r-1} v\right\}$ is equal to a Jordan block matrix.

Hence, it has only one eigenvalue $\lambda$ and one-dimensional eigenspace.

Definition 2.7 Fan and Fan basis By a fan of linear map A in vector space $V$, we shall mean a sequence of subspaces $\left\{V_{1}, \cdots, V_{n}\right\}$ such that $V_{i}$ is contained in $V_{i+1}$ for each $i=1, \cdots, n-1$, such that $\operatorname{dim} V_{i}=i$, and finally such that each $V_{i}$ is $(\boldsymbol{A}-\alpha \boldsymbol{I})$-invariant. By a fan basis, we shall mean a basis $\left\{v_{1}, \cdots, v_{n}\right\}$ of $V$ such that $\left\{v_{1}, \cdots, v_{i}\right\}$ is a basis for $V_{i}$.

\section{Cross-Identity Theorem}

To state the results of this paper, some more notations need to be introduced. As is known, the customary eigenspace of a linear map $\boldsymbol{A} \in L(V)$ associated with eigenvalue $\lambda$ is equivalent to $\operatorname{Ker}(\boldsymbol{A}-\lambda \boldsymbol{I})$, and the corresponding generalized eigenspaces are the union set of $\operatorname{Ker}(\boldsymbol{A}-\lambda \boldsymbol{I})^{m}, m=2,3, \cdots$. For convenience, we refer to $\operatorname{Ker}\left(\boldsymbol{A}^{m}\right)$ and $\operatorname{Ima}\left(\boldsymbol{A}^{m}\right)$ as power-m kernel and power-m image with respect to linear map $\boldsymbol{A}$, respectively. In this case, the customary eigenspace and the generalized eigenspaces associated with eigenvalue $\lambda$ are the power-one kernel and the higher power kernels of linear map $\boldsymbol{A}-\lambda \boldsymbol{I}$. 
Since a vector space can be expressed as a direct sum of $\boldsymbol{A}$-invariant cyclic subspaces, we only consider one of its $(\boldsymbol{A}-\lambda \boldsymbol{I})$-cyclic subspace, or without loss of generality, suppose $V$ entirely is a $(\boldsymbol{A}-\lambda \boldsymbol{I})$-cyclic space hereafter. In this situation, according to Theorem 2.6, $\boldsymbol{A}$ has only one eigenvalue and one-dimensional eigensapce. In addition, according to the direct sum decomposition of the $n$-dimensional vector space, there shall be $V=\operatorname{Ker}(\boldsymbol{A}-\lambda \boldsymbol{I})^{n}$, which means $\boldsymbol{A}-\lambda \boldsymbol{I}$ is nilpotent on cyclic space $V$. In what follows, we will show step by step that in this situation, there is a concise relationship between the corresponding power kernels and power images of linear map $\boldsymbol{A}-\lambda \boldsymbol{I}$. For simplicity, the following abbreviations of notations are introduced:

$$
\begin{aligned}
K^{m} & \equiv \operatorname{Ker}(\boldsymbol{A}-\lambda \boldsymbol{I})^{m}, m=1,2, \cdots \\
\boldsymbol{I}^{m} & \equiv \operatorname{Ima}(\boldsymbol{A}-\lambda \boldsymbol{I})^{m}, m=1,2, \cdots \\
\bar{K}^{m} & \equiv \operatorname{Ker}(\boldsymbol{A}-\lambda \boldsymbol{I}) \mid I^{m}, m=1,2, \cdots \\
\bar{I}^{m} & \equiv \operatorname{Ima}(\boldsymbol{A}-\lambda \boldsymbol{I}) \mid I^{m}, m=1,2, \cdots
\end{aligned}
$$

where $\operatorname{Ker}(\boldsymbol{A}-\lambda \boldsymbol{I}) \mid I^{m}$ means the restricted eigenspace in subspace $I^{m}$, and $\operatorname{Ima}(\boldsymbol{A}-\lambda \boldsymbol{I}) \mid I^{m}$ means the restricted image of $A-\lambda I$ on $I^{m}$. In what follows, the power 1 will be omitted when $m=1$, i.e. $K=K^{1}$, $I=I^{1}, \bar{K}=\bar{K}^{1}, \bar{I}=\bar{I}^{1}$. Then it is easy to verify that $\bar{K}^{m} \subset K, \bar{I}^{m}=I^{m+1}, m=1,2, \cdots$. The above analysis also readily yields that $\operatorname{dim}(K)=1, K^{n}=V$.

Now, it is ready to present some further results for a cyclic space as follows.

Lemma 3.1 If $I^{m} \neq\{0\}$, then $K \subset I^{m}, m=1,2, \cdots$.

Proof Note that $I^{m}, m=1,2, \cdots$ are all $\boldsymbol{A}$-invariant subspaces, then since $I^{m} \neq 0, \boldsymbol{A}$ must have an eigenvector in $I^{m}$, which implies $\operatorname{dim}\left(\bar{K}^{m}\right) \geq 1, m=1,2, \cdots$. Recall that $\bar{K}^{m} \subset K$ and $\operatorname{dim}(K)=1$, it leads straightforward to $K=\bar{K}^{m}$. Note $\bar{K}^{m} \subset I^{m}$, then we have $K \subset I^{m}$.

Lemma 3.2 If $p=\operatorname{dim}\left(I^{m}\right)>0$, then $\operatorname{dim}\left(I^{m+1}\right)=p-1, \quad m=1,2, \cdots$.

Proof $p=\operatorname{dim}\left(I^{m}\right)>0$ implies $I^{m} \neq\{0\}$, then from Lemma 3.1 there will be $\operatorname{dim}\left(\bar{K}^{m}\right)=1, m=1,2, \cdots$. Let $q=\operatorname{dim}\left(\bar{I}^{m}\right)=\operatorname{dim}\left(I^{m+1}\right)$, then it follows

$$
p=\operatorname{dim}\left(I^{m}\right)=\operatorname{dim}\left(\bar{K}^{m}\right)+\operatorname{dim}\left(\bar{I}^{m}\right)=1+q
$$

which results in $q=p-1$, i.e. $\operatorname{dim}\left(I^{m+1}\right)=p-1$.

From Lemma 3.2, one can see that for a cyclic subspace, the sequence of the dimensions of power images $I^{m}$ decrease with step one, and hence, the sequence of dimensions of power kernels $K^{m}$ will increase with step one.

Corollary $3.3 \operatorname{dim}\left(K^{m}\right)=m, \operatorname{dim}\left(I^{m}\right)=n-m, 1 \leq m \leq n$.

Proof Note that $\operatorname{dim}(K)=1$, there must be $\operatorname{dim}(I)=n-1$. By Lemma 3.2, it follows that $\operatorname{dim}\left(I^{m}\right)=n-m$. Since $\operatorname{dim}\left(I^{m}\right)+\operatorname{dim}\left(K^{m}\right)=n$, then we have $\operatorname{dim}\left(K^{m}\right)=m$.

Theorem 3.4 (Cross-Identity Theorem) The power kernels and power images of linear map $A$ on a cyclic space have the following Cross-Identity relationship, i.e.

$$
K=I^{n-1}, \cdots, K^{m}=I^{n-m}, \cdots, K^{n-1}=I
$$

Proof By Corollary 3.3, we can know readily that $K^{n}=V, I^{n}=\{0\}$. Moreover, according to the definition of $I^{m}, K^{m}$, it is easy to verify the following inclusion sequence.

$$
\begin{gathered}
K \subset K^{2} \subset \cdots \subset K^{n-1} \subset K^{n}=V \\
I \supset I^{2} \supset \cdots \supset I^{n-1} \supset I^{n}=\{0\}
\end{gathered}
$$

We give the proof in what follows by two steps. The first step is to prove $K^{n-1}=I$. Since $\operatorname{dim}(I)=\operatorname{dim}\left(K^{n-1}\right)=n-1$, so for $\forall y \in I$, there is a $x \in V$ such that $(\boldsymbol{A}-\lambda \boldsymbol{I}) x=y$, which yields $(A-\lambda I)^{n} x=(A-\lambda I)^{n-1} y=0$. Hence, $y \in K^{n-1}$, and therefore $I \subset K^{n-1}$. Because $I$ and $K^{n-1}$ have the same dimension, there must be $I=K^{n-1}$. Then we have $K \subset K^{2} \subset \cdots \subset K^{n-1}=I$. Next step is to prove $K^{m}=I^{n-m}, 1<m<n$. For $\forall x \in K^{m}$, we have $(A-\lambda I)^{m} x=0$. Since $m \leq n-1$, then $x \in K^{m} \subset K^{n-1}=I$. Hence, there must exist an $x_{1} \in V$ such that $(\boldsymbol{A}-\lambda \boldsymbol{I}) x_{1}=x$ with $(\boldsymbol{A}-\lambda \boldsymbol{I})^{m+1} x_{1}=0$. Since $m+1<n$, then 
$x_{1} \in K^{m+1} \subset I$, which means there must exit an $x_{2} \in V$ such that $(A-\lambda I) x_{2}=x_{1}$ with $(A-\lambda I)^{m+2} x_{2}=0$. Carrying on with this procedure, one can find an $x_{n-m}$ such that $(A-\lambda I) x_{n-m}=x_{n-m-1}$ with $(A-\lambda I)^{n} x_{n-m}=0$. Now it can be deduced from above that $(A-\lambda I)^{n-m} x_{n-m}=x$, i.e. $x \in I^{n-m}$, which implies $K^{m} \subset I^{n-m}$. According to Corollary 3.3, $\operatorname{dim}\left(K^{m}\right)=\operatorname{dim}\left(I^{n-m}\right)=m$, thus, we can readily contend that $K^{m}=I^{n-m}$

Corollary 3.5 Suppose matrix $\boldsymbol{A}$ has only one eigenvalue, and the corresponding eigenspace $v$ is one dimensional, then there must exist a transformation matrix $T$ such that $T^{-1} A T$ is a Jordan block matrix.

Proof Let matrix $\boldsymbol{A}$ be the associated matrix of a linear map $\boldsymbol{A} \in L(V)$ under a basis $\left\{\varepsilon_{1}, \varepsilon_{2}, \cdots \varepsilon_{n}\right\} \in V$. Since $\operatorname{dim}(I)=\operatorname{dim}\left(K^{n-1}\right)=n-1$, then there must be an $\alpha \in V$ such that $\alpha \notin K^{n-1}$, i.e. $(A-\lambda I)^{n-1} \alpha \neq 0$. Let $\eta_{1}=(\boldsymbol{A}-\lambda \boldsymbol{I})^{n-1} \alpha, \eta_{2}=(\boldsymbol{A}-\lambda \boldsymbol{I})^{n-2} \alpha, \cdots, \eta_{n-1}=(\boldsymbol{A}-\lambda \boldsymbol{I}) \alpha, \eta_{n}=\alpha$, then it is easy to verify that $\left\{\eta_{1}, \eta_{2}, \cdots \eta_{n}\right\}$ constitutes a fan basis of $V$. Thus, it is available to define an invertible linear transformation $\boldsymbol{T}\left(\varepsilon_{j}\right)=\eta_{j}$ with an invertible transformation matrix $T$, such that

$$
\left(\eta_{1}, \eta_{2}, \cdots, \eta_{n}\right)=\left(\varepsilon_{1}, \varepsilon_{2}, \cdots, \varepsilon_{n}\right) T
$$

Notice that $\eta_{j}=(\boldsymbol{A}-\lambda \boldsymbol{I})^{n-j} \alpha$, we have $(\boldsymbol{A}-\lambda \boldsymbol{I}) \eta_{j}=(\boldsymbol{A}-\lambda \boldsymbol{I})^{n-(j-1)} \alpha=\eta_{j-1}$, i.e. $\boldsymbol{A} \eta_{j}=\eta_{j-1}+\lambda \eta_{j}$. Then, $\boldsymbol{T}^{-1} \boldsymbol{A} \boldsymbol{T}\left(\varepsilon_{j}\right)=\boldsymbol{T}^{-1} \boldsymbol{A} \eta_{j}=\boldsymbol{T}^{-1}\left(\eta_{j-1}+\lambda \eta_{j}\right)=\varepsilon_{j-1}+\lambda \varepsilon_{j}$. From the above transformation we can contend that $T^{-1} A T$ is a Jordan block matrix with the following form

$$
\left(\begin{array}{lllll}
\lambda & 1 & 0 & \cdots & 0 \\
0 & \lambda & 1 & \cdots & 0 \\
\vdots & \vdots & \ddots & 1 & \vdots \\
0 & 0 & \cdots & \lambda & 1 \\
0 & 0 & \cdots & 0 & \lambda
\end{array}\right)
$$

\section{Conclusion}

This paper studies some special properties of cyclic subspaces. Although it is well known that the dimensions of the power kernels of a linear map on a vector space are decreasing, and the corresponding power images are increasing, i.e. $K \subset K^{2} \subset \cdots \subset K^{n}$ and $I \supset I^{2} \supset \cdots \supset I^{n}$, we show in this paper that for a cyclic subspace, the sequence of the dimensions of power kernels increases with step one, and the sequence of dimensions of power images decreases with step one. Besides, we find a Cross-Identity relationship between the power kernels and the power images, which is peculiar to cyclic subspaces. Another contribution of this paper is that we find a new way, by the application of the cross-identity theorem, to prove that a square matrix with only one eigenvalue and one-dimensional eigenspace is similar to a Jordan block matrix.

\section{Acknowledgements}

This work is supported by the National Natural Science Foundation of China (Grant No. 11172126). We also would like to thank the referees for their comments and suggestions.

\section{References}

[1] Hirsch, M.W., Smale, S. and Devaney R.L. (2004) Differential Equations, Dynamical Systems, and an Introduction to Chaos. 2nd Edition, Elsevier Academic Press, San Diego.

[2] Greub, W.H. (1967) Linear Algebra. 3rd Edition, Springer, Berlin.

[3] Lancaster, P. and Tismenetsky, M. (1985) The Theory of Matrices: With Applications. Academic Press Inc., San Diego.

[4] Lang, S. (1987) Linear Algebra. 3rd Edition, Springer, New York. http://dx.doi.org/10.1007/978-1-4757-1949-9

[5] Axler, S. (1997) Linear Algebra Done Right. Springer, New York. http://dx.doi.org/10.1007/b97662

[6] Lax, P.D. (2007) Linear Algebra and Its Applications. 2nd Edition, John Wiley \& Sons Inc, Hoboken, New Jersey.

[7] Roman, S. (2008) Advanced Linear Algebra. 3rd Edition, Springer, New York.

[8] Xu, Y.C. (2008) Linear Algebra and Matrix Theory. 2nd Edition, High Education Press, Beijing (in Chinese). 